\title{
Can axillary surgery be omitted in patients with breast pathologic complete response after neoadjuvant systemic therapy for breast cancer? A real-world retrospective study in China
}

\section{Rui Chen}

Jiangsu Province Hospital and Nanjing Medical University First Affiliated Hospital

\section{Shuo Li}

Jiangsu Province Hospital and Nanjing Medical University First Affiliated Hospital

\section{Yan Li}

Jiangsu Province Hospital and Nanjing Medical University First Affiliated Hospital

Qiannan Zhu

Jiangsu Province Hospital and Nanjing Medical University First Affiliated Hospital

\section{Xiaoqing Shi}

Jiangsu Province Hospital and Nanjing Medical University First Affiliated Hospital

Lu Xu

Jiangsu Province Hospital and Nanjing Medical University First Affiliated Hospital

\section{Yinggang $\mathrm{Xu}$}

Jiangsu Province Hospital and Nanjing Medical University First Affiliated Hospital

Weiwei Zhang

Jiangsu Province Hospital and Nanjing Medical University First Affiliated Hospital

Xiaofeng Huang

Jiangsu Province Hospital and Nanjing Medical University First Affiliated Hospital

\section{Jue Wang}

Jiangsu Province Hospital and Nanjing Medical University First Affiliated Hospital

Xiaoming Zha ( $\nabla$ njzhaxm@njmu.edu.cn )

Jiangsu Province Hospital and Nanjing Medical University First Affiliated Hospital https://orcid.org/0000-0003-2604-811X

\section{Research Article}

Keywords: breast cancer, neoadjuvant systemic therapy, pathologic complete response, axillary surgery, Sentinel lymph node biopsy

Posted Date: July 22nd, 2021 
DOI: https://doi.org/10.21203/rs.3.rs-142860/v2

License: (c) (1) This work is licensed under a Creative Commons Attribution 4.0 International License. Read Full License

Version of Record: A version of this preprint was published at Journal of Cancer Research and Clinical Oncology on August 16th, 2021. See the published version at https://doi.org/10.1007/s00432-021-037638. 


\section{Abstract \\ Purpose}

Studies show that axillary surgery can be potentially omitted in certain breast cancer patients who achieve breast pathologic complete response (pCR) after neoadjuvant systemic therapy (NST). However, potential differences between the ypT0 and ypTis subgroups remain to be explored. Furthermore, whether axillary surgery can be omitted in patients with clinically assessed positive axillary lymph nodes $(\mathrm{cN}+)$ remains unknown. This study was to evaluate the status of axillary lymph nodes for patients who achieved breast pCR after NST in the real-world study.

\section{Methods}

This retrospective cohort study included 258 patients with early or locally advanced breast cancer who underwent breast and axillary surgery after NST. Clinical and pathologic data were compared between patients with breast pCR (ypT0/is) and those without breast pCR.

\section{Results}

The rate of breast pCR after NST was $27.1 \%$ (70/258). Among the patients with initial cN0, the rate of axillary pCR was similar between the breast pCR and breast non-pCR groups $(100 \%$ vs. $85.7 \%, P=$ 0.1543 ). Among those with breast PCR, the rate of axillary pCR was $100 \%$ in both the ypT0 and ypTis subgroups. Furthermore, among those with initial $\mathrm{CN}+$, the rate of axillary $\mathrm{pCR}$ was higher in the breast pCR group than in the breast non-pCR group $(82.7 \%$ vs. $22.9 \%, P<0.0001)$. Among the patients with breast pCR, the rate of axillary pCR was higher in the ypT0 subgroup than in the ypTis subgroup ( $94.3 \%$ vs. $58.8 \%, P=0.0034)$.

\section{Conclusion}

Axillary surgery may potentially be omitted in patients with initial cNO who achieve breast pCR (ypT0/is), and may also be considered for omission in patients with initial $\mathrm{cN}+$ who achieve ypT0 (not ypTis).

\section{Introduction}

Neoadjuvant systemic therapy (NST) is increasingly used in breast cancer treatment, not only for locally advanced disease but also for operable cases. Pathologic complete response (pCR) has been reported to be significantly associated with favorable disease-free and overall survival rates ${ }^{1,2}$. Therefore, considerable effort has been made to de-escalate therapies for patients with $\mathrm{pCR}$, as redundant therapies induce unnecessary side effects and affect patients' quality of life. In the WSG-ADAPT-TN trial, patients who achieved pCR after nanoparticle albumin-bound paclitaxel-based NST could skip postoperative 
anthracycline-containing chemotherapy for chemotherapy de-escalation ${ }^{3}$. Another aim is to de-escalate or omit surgery in patients with pCR. Results from a feasibility trial by the MD Anderson Cancer Center (MDACC) demonstrated a very high accuracy rate between extensive vacuum-assisted core biopsy/fineneedle aspiration biopsy and lumpectomy of the marked breast tumor bed after $\mathrm{NST}^{4}$, suggesting that breast surgery may be omitted in patients with documented breast pCR.

These accumulating data raise the possibility that surgery of axillary lymph nodes might be omitted. Generally, axillary lymph node surgery following NST includes sentinel lymph node biopsy (SNB) and axillary lymph node dissection (ALND). The results of the NSABP B-32 trial have demonstrated that ALND can be de-escalated to SNB after NST in patients with clinically assessed negative axillary lymph nodes (cN0), because SNB is effective and safe for this subpopulation ${ }^{5}$. However, performing SNB following NST in patients with clinically assessed positive axillary lymph nodes $(\mathrm{cN}+)$ is controversial. Several large clinical trials have demonstrated that SNB has a relatively high false negative rate in this subpopulation ${ }^{6-8}$. Therefore, ALND remains the standard surgical approach for patients with initial cN+.

On the other hand, evidence suggest that axillary lymph node status after NST reflects prognosis more accurately than the initial axillary status before $\mathrm{NST}^{9}$. Patients with axillary $\mathrm{pCR}$ have higher recurrencefree and overall survival rates compared with those with residual axillary disease ${ }^{10}$. In fact, about 22$35 \%$ of patients with $\mathrm{cN}+$ achieve axillary $\mathrm{pCR}^{8,11}$. Successful distinction of these patients after NST might facilitate the omission of axillary surgeries including ALND and SNB, thereby avoiding postoperative morbidities, such as lymphedema, arm pain, and reduced arm movement, as well as quality of life issues ${ }^{12}$.

Several studies have already shown that axillary lymph node status is highly associated with breast $\mathrm{pCR}^{1,9,13}$. The results of a single institution study from the MDACC which enrolled patients with human epidermal growth factor receptor 2 (HER2)-positive and triple-negative (TN) breast cancer revealed that all patients with initial cNO who achieved the ypTO status (no residual invasive or in situ carcinoma in the breast) also achieved axillary $\mathrm{pCR}$, indicating that omitting axillary surgery might be possible in these patients ${ }^{1}$. Subsequently, a similar study reviewing data from the National Cancer Database (NCDB) reported that $98.2 \%$ patients with initial cNO achieved axillary pCR if they achieved the status of no residual invasive carcinoma in the breast (ypT0/is) ${ }^{13}$. Meanwhile, less than $90 \%$ of patients with initial cN1 who achieved ypT0/is could achieve axillary PCR in the same study. These results indicate several possibilities. First, both studies focused mainly on HER2-positive and TN breast cancer and more evidence on other molecular subtypes of breast cancer is needed. Second, the definition of breast PCR was different between the two studies, wherein ypT0 and ypT0/is were used in the MDACC and NCDB studies, respectively. Therefore, potential differences between the ypT0 and ypTis subgroups should be clarified. Third, these studies enrolled patients with cN1 disease. In real-world practice, operable Chinese patients usually receive surgery first unless they have more advanced breast cancer such as lymph node metastases $(\mathrm{cN}+)$. Therefore, whether axillary surgery can be omitted in patients with $\mathrm{cN}+$ remains an important question. 
We therefore conducted a real-world study by the retrospective review of data from a breast cancer center. All molecular subtypes of locally advanced breast cancer with the status of $\mathrm{cNO}$ or $\mathrm{cN}+$ were analyzed to identify subgroups of patients who could potentially avoid axillary surgery after NST.

\section{Patients And Methods}

This retrospective cohort study at a single institution included the review of all clinical and pathologic data of patients with early or locally advanced breast cancer $\left(\mathrm{cT}_{1-4} \mathrm{~N}_{0-3} \mathrm{M}_{0}\right)$ who underwent breast and axillary surgery after NST between January 2015 and July 2019. All patients underwent clinical disease staging with physical examination as well as ultrasonography and/or magnetic resonance imaging of the breast and axilla before NST. Clinically assessed normal axillary lymph nodes were defined as cNO based on these evaluations. Specifically, all suspicious lymph nodes were evaluated by specimens obtained using fine-needle aspiration or core needle biopsy and those with no tumor cells were classified as cNO; those with tumor cells were classified as $\mathrm{cN}+$. Breast pCR was defined as the absence of invasive carcinoma in the breast, including ypT0 (no residual invasive or in situ carcinoma in the breast) and ypTis (in situ carcinoma in the breast) as the final pathologic results. Axillary pCR, i.e., ypNO, was defined as the absence of residual carcinoma including macrometastatic and micrometastatic axillary nodal disease, in axillary lymph nodes after NST. All axillary nodes were assessed by standard hematoxylin-eosin staining, and immunohistochemistry was performed if suspicious cells were identified.

Lesions with at least $1 \%$ of cells positive for estrogen receptor and/or progesterone receptor were classified as hormone receptor (HR)-positive, and those with $3+$ HER2 staining intensity by immunohistochemistry or with HER2 gene amplification by fluorescence in situ hybridization were classified as HER2-positive. Most of the patients received anthracycline- and taxane-based neoadjuvant chemotherapy, and patients with HER2-positive breast cancer also received trastuzumab. All patients underwent standard breast and axillary surgery after NST, and the final surgical approach was left to the surgeon's discretion and the patient's preference based on the advantages and disadvantages of each type of surgery.

For statistical analyses, descriptive variables were presented as means \pm standard deviation. Pathologic axillary status was calculated for patients with and without breast $\mathrm{PCR}$ and compared using relative risk ratios (RRs) with 95\% confidence intervals (Cls). Categorical variables were compared using the two-side chi-squared test. A $P<0.05$ was considered to indicate statistical significance. All statistical analyses were performed by SPSS (version 20.0).

\section{Results}

A total of 258 consecutive female patients with breast cancer who received NST followed by surgery were included in the present study. The mean age at diagnosis was 50 (range, 20-75) years, $53.9 \%(139 / 258)$ of the patients were under 50 years of age, and $53.1 \%$ (137/258) of the patients were premenopausal. Before NST, $70.2 \%(181 / 258)$ and $18.2 \%(47 / 258)$ of the patients had cT2 and cT3 breast cancer, 
respectively, and $79.5 \%(205 / 258)$ of the patients had cN+. Furthermore, the rates of patients with HRpositive/HER2-negative, HR-positive/HER2-positive, HR-negative/HER2-positive, and TN breast cancer were $40.3 \%$ (104/258), 16.3\% (42/258), 20.9\% (54/258), and 22.5\% (58/258), respectively. Regarding surgical approaches, most patients $(91.9 \%, 237 / 258)$ chose mastectomy and ALND was performed in $88 \%(227 / 258)$ of the patients. Breast pCR was achieved in $27.1 \%(70 / 258)$ of the patients, including those with ypT0 $(18.2 \%, 47 / 258)$ and ypTis $(8.9 \%,(23 / 258)$. Most patients $(60.1 \%, 155 / 258)$ achieved pathologic partial response. In addition, axillary pCR was achieved in $48.8 \%(126 / 258)$ of the patients (Table 1). 
Table 1

General and clinical characteristics of all enrolled patients

Characteristics $(n=258)$

Age at diagnosis, years, mean (range)

Age at diagnosis, years

$\leq 50$

$>50$

Menopausal status at diagnosis

Premenopausal

Postmenopausal

Clinical assessment of tumor size before NST

cT1

cT2

cT3

cT4

Clinical assessment of axillary node stage before NST

$\mathrm{cNO}$

$\mathrm{cN}+$

Molecular subtype of breast cancer

HR-positive/HER2-negative

HR-positive/HER2-positive

HR-negative/HER2-positive

$\mathrm{TN}$

Type of breast surgery

Mastectomy

Abbreviations:

NST, neoadjuvant systemic therapy; cT, clinically assessed tumor grade before NST; cN, clinically assessed axillary lymph node stage before NST; HR, hormone receptor; HER2, human epidermal growth factor receptor 2; TN, triple-negative; pCR, pathologic complete response; ypT0, no residual invasive or in situ carcinoma in the breast; ypTis, in situ carcinoma in the breast; pPR, pathologic partial response; $\mathrm{pSD}$, pathologic stable disease; $\mathrm{pPD}$, pathologic progressive disease; $y \mathrm{pN}$, pathologic axillary lymph node stage after NST n (\%)

$50(20-75)$

$139(53.9)$

$119(46.1)$

$137(53.1)$

$121(46.9)$

14 (5.4)

181 (70.2)

47 (18.2)

$16(6.2)$

53 (20.5)

205 (79.5)

104 (40.3)

42 (16.3)

54 (20.9)

58 (22.5)

237 (91.9) 


\begin{tabular}{|c|c|}
\hline Characteristics $(n=258)$ & $\mathrm{n}(\%)$ \\
\hline Breast-conserving surgery & $21(8.1)$ \\
\hline \multicolumn{2}{|l|}{ Type of axillary surgery } \\
\hline Axillary lymph node dissection & $227(88.0)$ \\
\hline Sentinel node biopsy & $31(12.0)$ \\
\hline \multicolumn{2}{|l|}{ Breast pathology } \\
\hline pCR (урт0) & $47(18.2)$ \\
\hline pCR (ypTis) & $23(8.9)$ \\
\hline pPR & $155(60.1)$ \\
\hline pSD & $30(11.6)$ \\
\hline pPD & $3(1.2)$ \\
\hline \multicolumn{2}{|l|}{ Axillary lymph node pathology } \\
\hline ypNO & $126(48.8)$ \\
\hline ypN1 & $63(24.4)$ \\
\hline ypN2 & $46(17.8)$ \\
\hline ypN3 & $23(8.9)$ \\
\hline \multicolumn{2}{|l|}{ Abbreviations: } \\
\hline \multicolumn{2}{|c|}{$\begin{array}{l}\text { NST, neoadjuvant systemic therapy; cT, clinically assessed tumor grade before NST; cN, clinically } \\
\text { assessed axillary lymph node stage before NST; HR, hormone receptor; HER2, human epidermal } \\
\text { growth factor receptor 2; TN, triple-negative; PCR, pathologic complete response; ypT0, no residual } \\
\text { invasive or in situ carcinoma in the breast; ypTis, in situ carcinoma in the breast; pPR, pathologic } \\
\text { partial response; pSD, pathologic stable disease; pPD, pathologic progressive disease; ypN, pathologic } \\
\text { axillary lymph node stage after NST }\end{array}$} \\
\hline
\end{tabular}

There were no differences in age at diagnosis, menopausal status, tumor size, and axillary node status before NST between the breast pCR group, defined as patients with ypT0/is status, and the breast nonpCR group. The patients with HR-positive/HER2-negative breast cancer was less likely to achieve breast pCR (ypT0/is) than those with other molecular subtypes $(P<0.001)$ (Table 2$)$. 
Table 2

Comparisons between patients with breast pCR (ypT0/is) and non-pCR (non-ypT0/is)

\section{Characteristics}

ypT0/is non-ypTo/is $P$ value

$n(\%) \quad n(\%)$

Age at diagnosis, years

$\leq 50(n=139)$

$36(25.9) \quad 103(74.1)$

0.6746

$>50(\mathrm{n}=119)$

$34(28.6) \quad 85(71.4)$

Menopausal status at diagnosis

Premenopausal $(n=137)$

$36(26.3) \quad 101(73.7)$

0.7800

Postmenopausal $(n=121)$

$34(28.1) \quad 87(71.9)$

Clinical assessment of tumor size before NST

cT1 $(n=14)$

$5(35.7) \quad 9(64.3)$

0.8966

cT2 $(n=181)$

$48(26.5) \quad 133(73.5)$

cT3 $(n=47)$

$13(27.7) \quad 34(72.3)$

cT4 $(n=16)$

$4(25.0)$

$12(75.0)$

Clinical assessment of axillary node stage before NST

$\mathrm{cN} 0(\mathrm{n}=53)$

$18(34.0) \quad 35(66.0)$

0.2271

$\mathrm{cN}+(\mathrm{n}=205)$

$52(25.4) \quad 153(74.6)$

Molecular subtype of breast cancer

HR-positive/HER2-negative $(n=104)$

8 (7.7)

$96(92.3)$

$<0.001^{\star}$

HR-positive/HER2-positive $(n=42)$

15 (35.7) 27 (64.3)

HR-negative/HER2-positive $(n=54)$

$27(50.0) \quad 27(50.0)$

$\mathrm{TN}(\mathrm{n}=58)$

$20(34.5) \quad 38$ (65.5)

Abbreviations: breast pCR (ypT0/is), no residual invasive carcinoma in the breast; breast non-pCR (non-ypT0/is), presence of residual invasive carcinoma in the breast; NST, neoadjuvant systemic therapy; cT, clinically assessed tumor grade before NST; cN, clinically assessed axillary lymph node stage before NST; HR, hormone receptor; HER2, human epidermal growth factor receptor 2; TN, HRnegative and HER2-negative

${ }^{*} P<0.05$

In the group of patients with initial cN0, the postoperative pathological examination of axillary lymph nodes showed that the rate of axillary pCR was similar between the breast PCR $(100 \%, 18 / 18)$ and breast non-pCR $(85.7 \%, 30 / 35, P=0.1543)$ subgroups. Among those with breast $\mathrm{pCR}$, the rates of axillary $\mathrm{pCR}$ 
were $100 \%$ for both the ypT0 and ypTis subgroups. Similar findings were found by the comparison of different molecular subtypes (Table 3). 
Table 3

Postoperative pathology of axillary lymph nodes in patients with initial cNO who achieved breast pCR (ypT0/is) and non-pCR (non-ypT0/is)

\begin{tabular}{|c|c|c|c|c|c|}
\hline \multirow[t]{2}{*}{ cNO patients } & \multicolumn{5}{|c|}{$\begin{array}{l}\text { Pathologically positive axillary lymph nodes } \\
\text { n (\%) }\end{array}$} \\
\hline & 0 & 1 & 2 & $\geq 3$ & Total \\
\hline \multicolumn{6}{|l|}{ Total } \\
\hline урто & $12(100)$ & 0 & 0 & 0 & $12(100)$ \\
\hline ypTis & $6(100)$ & 0 & 0 & 0 & $6(100)$ \\
\hline ypT0/is & $18(100)$ & 0 & 0 & 0 & $18(100)$ \\
\hline non-ypTo/is & $30(85.7)$ & $2(5.7)$ & $1(2.9)$ & $2(5.7)$ & $35(100)$ \\
\hline \multicolumn{6}{|c|}{ HR-positive/HER2-negative } \\
\hline урто & $1(100)$ & 0 & 0 & 0 & $1(100)$ \\
\hline ypTis & $2(100)$ & 0 & 0 & 0 & $2(100)$ \\
\hline yрт0/is & $3(100)$ & 0 & 0 & 0 & $3(100)$ \\
\hline non-ypT0/is & $15(75)$ & $2(10)$ & $1(5)$ & $2(10)$ & $20(100)$ \\
\hline \multicolumn{6}{|c|}{ HR-positive/HER2-positive } \\
\hline урт0 & 0 & 0 & 0 & 0 & 0 \\
\hline ypTis & $1(100)$ & 0 & 0 & 0 & $1(100)$ \\
\hline ypTo/is & $1(100)$ & 0 & 0 & 0 & $1(100)$ \\
\hline non-ypTo/is & $3(100)$ & 0 & 0 & 0 & $3(100)$ \\
\hline \multicolumn{6}{|c|}{ HR-negative/HER2-positive } \\
\hline урт0 & $4(100)$ & 0 & 0 & 0 & $4(100)$ \\
\hline ypTis & $3(100)$ & 0 & 0 & 0 & $3(100)$ \\
\hline ypT0/is & $7(100)$ & 0 & 0 & 0 & $7(100)$ \\
\hline non-ypT0/is & $4(100)$ & 0 & 0 & 0 & $4(100)$ \\
\hline TN & & & & & \\
\hline
\end{tabular}

Abbreviations: breast pCR (ypT0/is), no residual invasive carcinoma in the breast; breast non-pCR (non-ypT0/is), presence of residual invasive carcinoma in the breast; ypT0, no residual invasive or in situ carcinoma in the breast; ypTis, in situ carcinoma in the breast; $\mathrm{CN}$, clinically assessed axillary lymph node stage before NST; HR, hormone receptor; HER2, human epidermal growth factor receptor 2; TN, HR-negative and HER2-negative. 


\begin{tabular}{|c|c|c|c|c|c|}
\hline \multirow[t]{2}{*}{ cNO patients } & \multicolumn{5}{|c|}{$\begin{array}{l}\text { Pathologically positive axillary lymph nodes } \\
\text { n (\%) }\end{array}$} \\
\hline & 0 & 1 & 2 & $\geq 3$ & Total \\
\hline урТ0 & $7(100)$ & 0 & 0 & 0 & $7(100)$ \\
\hline ypTis & 0 & 0 & 0 & 0 & 0 \\
\hline урт0/is & $7(100)$ & 0 & 0 & 0 & $7(100)$ \\
\hline non-ypT0/is & $8(100)$ & 0 & 0 & 0 & $8(100)$ \\
\hline \multicolumn{6}{|c|}{$\begin{array}{l}\text { Abbreviations: breast pCR (ypT0/is), no residual invasive carcinoma in the breast; breast non-pCR } \\
\text { (non-ypT0/is), presence of residual invasive carcinoma in the breast; ypT0, no residual invasive or in } \\
\text { situ carcinoma in the breast; ypTis, in situ carcinoma in the breast; cN, clinically assessed axillary } \\
\text { lymph node stage before NST; HR, hormone receptor; HER2, human epidermal growth factor receptor } \\
\text { 2; TN, HR-negative and HER2-negative. }\end{array}$} \\
\hline
\end{tabular}

The postoperative pathological examination of axillary lymph nodes in patients with initial $\mathrm{cN}+$ revealed that the rate of axillary $\mathrm{pCR}$ was higher in the breast $\mathrm{pCR}$ subgroup compared with the breast non-pCR group (82.7\% [43/52] vs. $22.9 \%$ [35/153], $P<0.0001$, RR $2.520,95 \% \mathrm{Cl} 2.634-4.961)$. In the TN breast cancer subgroup, the rate of axillary pCR was $92.3 \%$ (12/13). Among the patients with breast pCR, the rate of axillary pCR in the ypT0 subgroup $(94.3 \%, 33 / 35)$ was higher than that in the ypTis subgroup $(58.8 \%, 10 / 17, P=0.0034, \mathrm{RR} 1.603,95 \% \mathrm{Cl} 1.068-2.406)$ (Table 4). 
Table 4

Postoperative pathology of axillary lymph nodes in patients with initial $\mathrm{CN}+$ who achieved breast $\mathrm{pCR}$ (ypT0/is) and non-pCR (non-ypT0/is)

\begin{tabular}{|c|c|c|c|c|c|}
\hline \multirow[t]{3}{*}{$\mathrm{cN}+$ patients } & \multicolumn{5}{|c|}{ Pathologically positive axillary lymph nodes } \\
\hline & \multicolumn{5}{|l|}{ No. (\%) } \\
\hline & 0 & 1 & 2 & $\geq 3$ & Total \\
\hline \multicolumn{6}{|l|}{ Total } \\
\hline урт0 & $33(94.3)$ & 0 & $1(2.9)$ & $1(2.9)$ & $35(100)$ \\
\hline ypTis & $10(58.8)$ & $3(17.6)$ & $1(5.9)$ & $3(17.6)$ & $17(100)$ \\
\hline ypT0/is & $43(82.7)$ & $3(5.8)$ & $2(3.8)$ & $4(7.7)$ & $52(100)$ \\
\hline non-ypTo/is & $35(22.9)$ & $23(15.0)$ & $17(11.1)$ & $78(51)$ & $153(100)$ \\
\hline \multicolumn{6}{|c|}{ HR-positive/HER2-negative } \\
\hline урт0 & $3(75)$ & 0 & $1(25)$ & 0 & $4(100)$ \\
\hline ypTis & $1(100)$ & 0 & 0 & 0 & $1(100)$ \\
\hline ypT0/is & $4(80)$ & 0 & $1(20)$ & 0 & $5(100)$ \\
\hline non-ypT0/is & $9(11.8)$ & $13(17.1)$ & $9(11.8)$ & $45(59.2)$ & $76(100)$ \\
\hline \multicolumn{6}{|c|}{ HR-positive/HER2-positive } \\
\hline урто & $6(100)$ & 0 & 0 & 0 & $6(100)$ \\
\hline ypTis & $4(50)$ & $2(25)$ & $1(12.5)$ & $1(12.5)$ & $8(100)$ \\
\hline ypT0/is & $10(71.4)$ & $2(14.3)$ & $1(7.1)$ & $1(7.1)$ & $14(100)$ \\
\hline non-ypTo/is & $6(25)$ & $5(20.8)$ & $1(4.2)$ & $12(50)$ & $24(100)$ \\
\hline \multicolumn{6}{|c|}{ HR-negative/HER2-positive } \\
\hline урт0 & $13(92.9)$ & 0 & 0 & $1(7.1)$ & $14(100)$ \\
\hline ypTis & $4(66.7)$ & $1(16.7)$ & 0 & $1(16.7)$ & $6(100)$ \\
\hline ypT0/is & $17(85)$ & $1(5)$ & 0 & $2(10)$ & $20(100)$ \\
\hline non-ypT0/is & $10(43.5)$ & $2(8.7)$ & $4(17.4)$ & $7(30.4)$ & $23(100)$ \\
\hline TN & & & & & \\
\hline
\end{tabular}

Abbreviations: breast pCR (ypT0/is), no residual invasive carcinoma in the breast; breast non-pCR (non-ypT0/is), presence of residual invasive carcinoma in the breast; ypT0, no residual invasive or in situ carcinoma in the breast; ypTis, in situ carcinoma in the breast; $\mathrm{CN}$, clinically assessed axillary lymph node stage before NST; HR, hormone receptor; HER2, human epidermal growth factor receptor 2; TN, HR-negative and HER2-negative 


\begin{tabular}{|c|c|c|c|c|c|}
\hline \multirow[t]{3}{*}{$\mathrm{cN}+$ patients } & \multicolumn{5}{|c|}{ Pathologically positive axillary lymph nodes } \\
\hline & \multicolumn{5}{|l|}{ No. (\%) } \\
\hline & 0 & 1 & 2 & $\geq 3$ & Total \\
\hline урт0 & $11(100)$ & 0 & 0 & 0 & $11(100)$ \\
\hline ypTis & $1(50)$ & 0 & 0 & $1(50)$ & $2(100)$ \\
\hline урт0/is & $12(92.3)$ & 0 & 0 & $1(7.7)$ & $13(100)$ \\
\hline non-ypT0/is & $10(33.3)$ & $3(10)$ & $3(10)$ & $14(46.7)$ & $30(100)$ \\
\hline \multicolumn{6}{|c|}{$\begin{array}{l}\text { Abbreviations: breast pCR (ypTO/is), no residual invasive carcinoma in the breast; breast non-pCR } \\
\text { (non-ypTO/is), presence of residual invasive carcinoma in the breast; ypTO, no residual invasive or in } \\
\text { situ carcinoma in the breast; ypTis, in situ carcinoma in the breast; cN, clinically assessed axillary } \\
\text { lymph node stage before NST; HR, hormone receptor; HER2, human epidermal growth factor receptor } \\
\text { 2; TN, HR-negative and HER2-negative }\end{array}$} \\
\hline
\end{tabular}

\section{Discussion}

Since the prognosis is good for both patients with ypTO ypNO and those with ypTis ypNO breast cancer ${ }^{14}$, breast PCR in the present study was defined as the absence of residual invasive carcinoma in the breast, including ypT0 and ypTis. Our analyses revealed that the rate of patients with ypT0/is ypNO after NST was $23.6 \%$, which was not high. There are several possibilities to explain this finding. First, the present study included all molecular subtypes of breast cancer and $40.3 \%$ of the patients had HR-positive/HER2negative breast cancer, who were less likely to achieve breast pCR (7.7\%) compared to those with other molecular subtypes $(P<0.01)$, especially compared to patients with HER2-positive $(43.8 \%)$ or TN $(34.5 \%)$ breast cancer. This finding is in agreement with other reports ${ }^{15,16}$. In addition, HER2-positive patients received trastuzumab only as targeted therapy in the present study, because pertuzumab was not approved for NST in China until 2020. Third, the tumor burden was greater in the current study cohort. The median tumor diameter was $50 \mathrm{~mm}, 24.4 \%$ of the patients were in stage cT3-4, and the rate of $\mathrm{cN}+$ was $79.5 \%$. These data reflect the real-world situation in China. Specifically, relatively more patients with advanced breast cancer have been selected to receive NST in recent years; however, those with operable breast cancer usually receive surgery first, even those patients with HER2-positive or TN breast cancer.

Due to the greater tumor burden, the rates of breast PCR were not high in the present study $(18.2 \%$ and $8.9 \%$ in the yрT0 and ypTis subgroups, respectively). On the other hand, a greater tumor burden was also associated with a higher rate of tumor metastasis to axillary lymph nodes and only $20.5 \%$ of the enrolled patients had cNO. After NST, however, the rate of axillary pCR increased to $48.8 \%$, indicating that NST could effectively reduce the stage of axillary lymph nodes and that nearly half of these patients may be able to avoid axillary surgery. Furthermore, determination of more accurate indicators can potentially facilitate the identification of patients who may avoid axillary surgery including ALND and SNB. Based on our analyses, the rate of patients achieving axillary $\mathrm{PCR}$ was higher in the breast pCR group than in the breast non-pCR group ( $87.1 \%$ vs $34.6 \%, P<0.01)$, indicating that those who achieved breast pCR were 
more likely to achieve axillary pCR. This finding suggests that breast PCR might be a good indicator to determine patients who might be able to avoid axillary surgery, which requires for exploration in future studies.

In the present study, 18 of the 53 patients with initial cNO achieved breast pCR after NST; all 18 patients $(100 \%)$ achieved axillary pCR simultaneously. In comparison, only 30 of the 35 patients with breast nonpCR (85.7\%) achieved axillary pCR. Although there was no statistical difference between the breast pCR and breast non-pCR groups, the high axillary pCR rate in the breast PCR group (100\%) provides evidence to support that axillary surgery may be omitted in patients with initial cNO who achieve breast $\mathrm{pCR}$. The study from the MDACC ${ }^{1}$ have also confirmed that axillary surgery might be omitted in patients with initial cNO who achieve ypTO. However, only patients with HER2-positive and TN breast cancer were enrolled and those with the ypTis status were not included in that study. In real-world settings, HR-positive/HER2negative breast cancer comprises the largest subtype despite the low rate of $\mathrm{pCR}$. Therefore, it is also especially important to explore whether axillary surgery can be omitted in patients with this breast cancer subtype. In the current study cohort, 104 (40.3\%) of the 258 enrolled patients were HR-positive/HER2negative, 23 of these 104 patients had cNO breast cancer, and 3 patients who achieved breast pCR also achieved axillary $\mathrm{pCR}$, with a success rate of $100 \%$. This finding may indicate that axillary surgery can also be potentially omitted in patients with HR-positive/HER2-negative breast cancer and initial cNO who achieve ypT0/is status, however, due to the small amount, this needs to be supported by larger data subsequently. This result is consistent with the analysis from other studies ${ }^{13,17}$, which also provides support that axillary surgery might be omitted in patients with initial cNO breast cancer of any molecular subtype who achieve the ypT0/is status.

On the other hand, 153 of the 205 patients with initial $\mathrm{cN}+$ achieved breast non-pCR after NST and only 35 of these patients $(22.9 \%)$ achieved axillary pCR. In comparison, the rate of axillary pCR was higher in patients who achieved breast pCR $(43 / 52,82.7 \%)(P<0.01)$. However, since the rate of axillary pCR is less than $90 \%$, axillary surgery omission is not safe in patients with initial $\mathrm{CN}+$ who achieve breast pCR. In addition, the subgroup analysis showed that only patients with TN breast cancer (12/13, 92.3\%) might potentially be considered for axillary surgery omission. This finding is also consistent with the report from the MDACC ${ }^{1}$ which demonstrated that the omission of axillary surgery might be safe for patients with TN breast cancer $(32 / 35,91.4 \%)$. However, the subgroup analysis in the NCDB study ${ }^{13}$ led to a different result, wherein the omission of axillary surgery was not safe for all molecular subtypes including TN breast cancer. This discrepancy may be due to differences in the characteristics of patients enrolled in different studies. Therefore, prospective multicenter clinical trials are necessary to obtain more accurate results.

Furthermore, we analyzed patients with initial $\mathrm{cN}+$ breast cancer by categorizing into the ypT0 and ypTis subgroups. Our analyses demonstrated that the axillary pCR rate was higher in the ypT0 subgroup than in the ypTis subgroup $(94.3 \%$ vs $58.8 \%, P<0.01)$. Meanwhile, the high rate value $(>90 \%)$ was consistent across all molecular subgroups except for the HR-positive/HER2-negative subgroup, in which only four 
patients achieved ypT0, indicating that axillary surgery may also be potentially omitted if ypT0, rather than ypTis, is achieved in patients with initial cN + HER2-positive or TN breast cancer.

The limitations of the present study include a small-sized cohort in a real-world study, which was conducted at a single breast cancer center. Additionally, due to the retrospective study design, the patients with $\mathrm{cN}+$ breast cancer could not be analyzed further to distinguish those with $\mathrm{cN} 1$ disease from those with $\mathrm{cN} 2$ or $\mathrm{cN} 3$ disease. However, based on the postoperative pathology, nearly $1 / 3$ of the patients were in ypN2-3, implying that many of the patients had advanced axillary tumor burden. Therefore, prospective multicenter clinical trials will be necessary to obtain more reliable evidence.

\section{Conclusion}

In the present study including patients with advanced breast cancer receiving NST in a real-world setting in line with the clinical reality in China, our analyses suggested that axillary surgery might be potentially omitted in patients with initial cNO breast cancer who achieve breast pCR (ypT0/is) regardless of the molecular subtype and clinical tumor size. Additionally, axillary surgery might also be potentially omitted in patients with initial $\mathrm{cN}+$ breast cancer who achieve ypT0 (not ypTis), excluding those with HRpositive/HER2-negative breast cancer.

\section{Declarations}

\section{Funding}

The author(s) received no financial support for the research, and/or publication of this article.

\section{Availability of data and material}

The datasets used and analyzed during the current study are available from the corresponding author on reasonable request.

\section{Conflict of interests}

The authors declare that they have no confict of interest.

\section{Acknowledgments}

This research was supported in part by Jiangsu Province Six Talents Summit Project (WSW-001), Youth Talent Project (FRC201308), Jiangsu Women and Children Health Research Project (F201761), Chinese Society of Clinical Oncology Foundation (Y-sy2018-077, Y-JS2019-096), National Natural Science Foundation of China (81302305), Natural Science Foundation of Jiangsu Province (BK20131027), Jiangsu Province Women and Children Health Scientific Research Project (F201821).

\section{Authors' contributions}


Xiaoming Zha, Jue Wang designed this study and conducted quality control on it. Rui Chen, Yan Li, Xiaoqing Shi participated in the analysis of data and drafted the manuscript. Shuo Li, Qiannan Zhu, Lu $\mathrm{Xu}$, Yinggang $\mathrm{Xu}$, Weiwei Zhang and Xiaofeng Huang were responsible for data collection. All authors read and approved the final manuscript.

\section{Ethics approval and consent to participate}

All procedures were followed in accordance with the ethical standards of the responsible committee on human experimentation (institutional and national) and those of the Helsinki Declaration. This work was approved by the ethics and research committee of the First Affiliated Hospital of Nanjing Medical University (2020-SR-081), and informed consent was obtained from all participants.

\section{References}

1. Tadros AB, Yang WT, Krishnamurthy S, Rauch GM, Smith BD, Valero V et al (2017) Identifcation of patients with documented pathologic complete response in the breast after neoadjuvant chemotherapy for omission of axillary surgery. JAMA Surg 152:665-670

2. Heil J, Kummel S, Schaefgen B, Paepke S, Thomssen C, Rauch G et al (2015) Diagnosis of pathological complete response to neoadjuvant chemotherapy in breast cancer by minimal invasive biopsy techniques. Br J Cancer 113:1565-1570

3. Gluz O, Nitz U, Liedtke C, Christgen M, Grischke EM, Forstbauer H et al (2018) Comparison of Neoadjuvant Nab-Paclitaxel + Carboplatin vs Nab-Paclitaxel + Gemcitabine in Triple-Negative Breast Cancer: Randomized WSG-ADAPT-TN Trial Results. J Natl Cancer Inst 110:628-637

4. Kuerer HM, Rauch GM, Krishnamurthy S, Adrada BE, Caudle AS, DeSnyder SM et al (2018) A clinical feasibility trial for identification of exceptional responders in whom breast cancer surgery can be eliminated following neoadjuvant systemic therapy. Ann Surg 267:946-951

5. Krag DN, Anderson SJ, Julian TB, Brown AM, Harlow SP, Costantino JP et al (2010) Sentinel-lymphnode resection compared with conventional axillary-lymph-node dissection in clinically nodenegative patients with breast cancer: overall survival findings from the NSABP B-32 randomised phase 3 trial. Lancet Oncol 11:927-933

6. Boughey JC, Suman VJ, Mittendorf EA, Ahrendt GM, Wilke LG, Taback B et al (2013) Sentinel lymph node surgery after neoadjuvant chemotherapy in patients with node-positive breast cancer: the ACOSOG Z1071 (Alliance) clinical trial. JAMA 310:1455-1461

7. Kuehn T, Bauerfeind I, Fehm T, Fleige B, Hausschild M, Helms G et al (2013) Sentinel-lymph-node biopsy in patients with breast cancer before and after neoadjuvant chemotherapy (SENTINA): a prospective, multicentre cohort study. Lancet Oncol 14:609-618

8. Shen J, Gilcrease MZ, Babiera GV, Ross MI, Meric-Bernstam F, Feig BW et al (2007) Feasibility and accuracy of sentinel lymph node biopsy after preoperative chemotherapy in breast cancer patients with documented axillary metastases. Cancer 109:1255-1263 
9. Choi HJ, Ryu JM, Kim I, Gil-Gil M, Perez-Montero H, Fernandez-Montolí E et al (2019) Prediction of axillary pathologic response with breast pathologic complete response after neoadjuvant chemotherapy. Breast Cancer Res Treat 176:591-596

10. Mougalian SS, Hernandez M, Lei X, Lynch S, Kuerer HM, Symmans WF et al (2016) Ten-year outcomes of patients with breast cancer with cytologically confirmed axillary lymph node metastases and pathologic complete response after primary systemic chemotherapy. JAMA Oncol $2: 508-516$

11. Hennessy BT, Hortobagyi GN, Rouzier R, Kuerer H, Sneige N, Buzdar AU et al (2005) Outcome after pathologic complete eradication of cytologically proven breast cancer axillary node metastases following primary chemotherapy. J Clin Oncol 23:9304-9311

12. Mansel RE, Fallowfield L, Kissin M, Goyal A, Newcombe RG, Dixon JM et al (2006) Randomized multicenter trial of sentinel node biopsy versus standard axillary treatment in operable breast cancer: the ALMANAC Trial. J Natl Cancer Inst 98:599-609

13. Barron AU, Hoskin TL, Day CN, Hwang ES, Kuerer HM, Boughey JC (2018) Association of low nodal positivity rate among patients with ERBB2-positive or triple-negative breast cancer and breast pathologic complete response to neoadjuvant chemotherapy. JAMA Surg 153:1120-1126

14. Kong X, Moran MS, Zhang N, Haffty B, Yang Q (2011) Meta-analysis confirms achieving pathological complete response after neoadjuvant chemotherapy predicts favourable prognosis for breast cancer patients. Eur J Cancer 47:2084-2090

15. von Minckwitz G, Untch M, Blohmer JU, Costa SD, Eidtmann H, Fasching PA et al (2012) Definition and impact of pathologic complete response on prognosis after neoadjuvant chemotherapy in various intrinsic breast cancer subtypes. J Clin Oncol 30:1796-1804

16. Houssami N, Macaskill P, von Minckwitz G, Marinovich ML, Mamounas E (2012) Meta-analysis of the association of breast cancer subtype and pathologic complete response to neoadjuvant chemotherapy. Eur J Cancer 48:3342-3354

17. amiei S, van Nijnatten TJA, de Munck L, Keymeulen KBMI, Simons JM, Kooreman LFS et al (2020) Correlation Between Pathologic Complete Response in the Breast and Absence of Axillary Lymph Node Metastases After Neoadjuvant Systemic Therapy. Ann Surg 271:574-580 